Pacific Journal of Mathematics

WHIRR OPERATORS I B BANACH PAC 


\section{UNITARY OPERATORS IN BANACH SPACES}

\section{T. V. PANCHAPAGESAN}

The notion of hermitian operators in Hilbert space has been extended to Banach spaces by Lumer and Vidav. Recently, Berkson has shown that a scalar type operator $S$ in a Banach space $X$ can be decomposed into $S=R+i J$ where (i) $R$ and $J$ commute and (ii) $R^{m} J^{n}(m, n=0,1,2, \cdots)$ are hermitian in some equivalent norm on $X$. The converse is also valid if the Banach space is reflexive. Thus we see that the scalar type operators in a Banach space play a role analogous to the normal operators in a Hilbert space.

In this paper, the well-known Hilbert space notion of unitary operators is suitably extended to operators in Banach spaces and a polar decomposition is obtained for a scalar type operator. It is further shown that this polar decomposition is unique and characterises scalar type operators in reflexive Banach spaces. Finally, an extension of Stone's theorem on one-parameter group of unitary operators in Hilbert spaces is obtained (under suitable conditions) for reflexive Banach spaces.

The terminology and notation in this paper are as follows. The term Banach space always means a complex Banach space. The Banach algebra of all operators on a Banach space $X$ is denoted by $B(X)$. For an operator $T$ on a Banach space $X$, the spectrum of $T$ is denoted by $\sigma(T)$. The term spectral operator on a Banach space $X$ refers to a spectral operator of class $X^{*}$. The exponential function at the operator $T$ is defined and denoted as below.

$$
e^{T}=\sum_{n=0}^{\infty} \frac{T^{n}}{n !} .
$$

Definition 1. An operator $T$ on a Banach space $X$ is said to be hermitian under the equivalent norm $\|\cdot\|$ on $X$, if $[T x, x]$ is real for all $x$ in $X$ with $\|x\|=1$, where | , ] is some semi-inner-product on $X$, inducing the norm $\|\cdot\|$.

The above definition is due to Lumer and in [7] it is shown that

(1.1) an operator $T$ is hermitian under the equivalent norm $\|\cdot\|$ on $X$ if and only if $\|I+i r T\|=1+o(r)$ for real $r$, as $r$ tends to zero.

Further as a consequence of Lemma 1 of Vidav [9], it follows that

(1.2) if $T$ is hermitian under the equivalent norm $\|\cdot\|$ on $X$, then $\left\|e^{i r T}\right\|=1$ for all real $r$. 
Definition 2. A family of operators on a Banach space $X$ is said to be hermitian-equivalent (after Lumer) if there exists an equivalent renorming of the space $X$, under which all the members of the given family become hermitian.

It is proved in [8] by Lumer ${ }^{1}$ :

(1.3) Suppose $F_{i} \quad i=1,2, \cdots, n$ are finitely many commuting families of operators on a Banach space $X$, each of them being hermitian-equivalent and $F=\bigcup_{i=1}^{n} F_{i}$ is also a commuting family. Then the family $F$ is hermitian-equivalent.

Definition 3. If $S$ is a scalar type operator on a Banach space, with $E($.$) as the resolution of the identity, then S=\int \lambda E(d \lambda)$. We call $\int \operatorname{Re} \lambda E(d \lambda)$ and $\int \operatorname{Im} \lambda E(d \lambda)$ the real and imaginary parts of $S$ and denote them by $\operatorname{Re} S$ and $\operatorname{Im} S$ respectively.

(1.4) If $S$ is a scalar type operator on a Banach space $X$ and $S=R+i J$ where $R$ and $J$ commute and $R$ and $J$ are hermitian in some equivalent norms on $X$, then $R=\operatorname{Re} S$ and $J=\operatorname{Im} S$. (See Lumer [8]).

The following two results are due to Foguel [4].

(1.5) Let $T$ be a spectral operator on a Banach space $X$. Then there exist two operators $P$ and $U$ on $X$ such that

(1) $T=P U=U P$;

(2) $\sigma(P)$ is nonnegative and $\sigma(U)$ is a subset of the unit circle;

(3) $U$ is scalar type and $P$ is spectral. Further, the conditions (1), (2) and (3) insure uniqueness of $P$ and $U$.

(1.6) A spectral operator $T$ on a Banach space $X$ is a scalar type operator with its spectrum lying on the unit circle if and only if $T^{-1}$ is a bounded everywhere defined operator on $X$ and there exists a constant $K$ such that

$$
\left\|T^{n}\right\| \leqq K \quad \text { for } n= \pm 1, \pm 2, \cdots .
$$

2. Definition of a unitary operator in a Banach space. In this section we give the definition of a unitary operator in a Banach space and make some observations.

Definition 4. A spectral operator $U$ on a Banach space $X$ is said to be unitary under the equivalent norm $\|\cdot\|$ on $X$, if and only

1 The author wishes to thank Prof. G. Lumer, for making available to him a pre-print of the latter's paper [8]. 
if $U$ is an isometry of $X$ onto itself under the norm $\| \cdot$

As shown by Fixman in [3], an onto isometry of a Banach space $X$ need not be spectral, even when $X$ is reflexive. However, if $X$ is a Hilbert space in the norm $\|\cdot\|$ and $U$ is an onto isometry of $X$ in this norm $\|\cdot\|$, then $U$ is spectral by classical Hilbert space spectral theory and hence $U$ is unitary in our sense also.

By applying the result (1.7) of $\S 1$, to the definition of a unitary operator, we have the following remark.

REMARK. If $U$ is a unitary operator under the equivalent norm $\|\cdot\|$ on $X$, then $U$ is scalar type and $\sigma(U)$ lies on the unit circle.

The converse of the above remark is also true which will be proved in $\S 3$.

3. Polar decomposition of a scalar type operator. We obtain here a polar decomposition of a scalar type operator on a Banach space analogous to that of a normal operator on a Hilbert space.

Definition 5. An operator $R$ on a Banach space $X$ is said to be positive under the equivalent norm $\|\cdot\|$, if $R$ is hermitian under the norm $\|\cdot\|$ and $\sigma(R)$ is nonnegative.

LEMmA 1. If a scalar type operator $S$ has its spectrum on the unit circle, then $T=\operatorname{Re} S$ and $T^{\prime}=\operatorname{Im} S$ satisfy the condition

$$
\left.T^{2}+T^{\prime 2}=I \text { (the identity operator }\right)
$$

Proof. This follows from the fact that for $\lambda$ on the unit circle $(\operatorname{Re} \lambda)^{2}+(\operatorname{Im} \lambda)^{2}=1$.

THEOREM 1. If $S$ is a scalar type operator on a Banach space $X$, then there exist operators $R$ and $U$ and an equivalent norm $\|\cdot \mid\|$ on $X$ such that

(i) $S=R U$ where $R$ is positive and $U$ is unitary under the norm $|\| \cdot|||$;

(ii) $T=\operatorname{Re} U, T^{\prime}=\operatorname{Im} U$ and $R$ form a commuting family of operators on $X$ such that $R^{m} T^{n} T^{\prime l}(m, n, l=0,1,2, \cdots)$ are hermitian under the norm ||$|\cdot| \mid$.

Further the above decomposition is unique.

Proof. First, we shall obtain the decomposition $S=R U$ with $R$ and $U$ satisfying conditions (i) and (ii) of the theorem, under an equivalent norm. If $E($.$) is the resolution of the identity of S$, then by Lemma 2, p. 60 of Foguel [4], $R=\int|\lambda| E(d \lambda)$ and 


$$
U=\int(\operatorname{sgn} \lambda) E(d \lambda)
$$

give a decomposition of $S$ with $\sigma(R)$ nonnegative and $\sigma(U)$ as a subset of the unit circle. Define

$$
\begin{aligned}
& \|x\| \mid=\sup \operatorname{var} x^{*} E(\cdot) x \\
& \|x\| \mid=\sup _{\left\|x^{*}\right\|=1} \operatorname{var} x^{*} E(\cdot) x
\end{aligned}
$$

where $\|\cdot\|$ denotes the given norm of the Banach space $X$. Then, as proved by Berkson [1], $\||\cdot| \mid$ is an equivalent norm on $X$ and with respect to this norm $\|\cdot \cdot\|, E(\sigma)$ are hermitian for Borel sets $\sigma$ of the complex plane. That $R$ is positive in $\||\cdot|\|$ is clear. Since the operator $U$ is scalar type with its spectrum lying on the unit circle, $U$ takes the form $U=e^{i K}$, where $K$ is the scalar type operator

$$
K=\int_{0}^{2 \pi} \theta F(d \theta)
$$

with its resolution of the identity $F($.$) given by$

$$
F(\sigma)=E\left\{e^{i \theta}: \theta \in \sigma \cap(0,2 \pi]\right\}
$$

for Borel sets $\sigma$ of the complex plane. Since $E(\sigma)$ and hence $F(\sigma)$ are hermitian in $\mid\|\cdot\|, K$ is hermitian in $\|\cdot \mid\|$ and therefore we have ||$|U|\left\|=||\left|e^{i \Sigma}\right|\right\|=1$ by (1.2) of $\S 1$. Similarly, ||$U^{-1}|| \mid=1$ so that $U$ is an onto isometry in $\|\cdot\| \|$. Hence $U$ is unitary in $\|\cdot\| \|$. It is clear that $R, T$ and $T^{\prime}$ satisfy condition (ii) if one observes that

$$
T=\int \operatorname{Re}(\operatorname{sgn} \lambda) E(d \lambda) \text { and } T^{\prime}=\int \operatorname{Im}(\operatorname{sgn} \lambda) E(d \lambda) .
$$

Now we shall show that the above decomposition of $S$ is unique. If possible, let $S=R_{1} U_{1}$ where $R_{1}$ and $U_{1}$ satisfy conditions (i) and (ii) under an equivalent norm $\|\cdot\|_{1}$ (say). To show $R_{1}=R$ and $U_{1}=U$ (see (1) for the definition of $U$ and $R$ ), in view of (1.5) of $\S 1$ it suffices to show that $R_{1}$ is spectral. Now we will prove ${ }^{2}$ that $R_{1}=R$, so that $R_{1}$ is spectral. If $T_{1}=\operatorname{Re} U_{1}$ and $T_{1}^{\prime}=\operatorname{Im} U_{1}$, then $S=R_{1} T_{1}+i R_{1} T_{1}^{\prime}$. Now by assumption, $R_{1} T_{1}$ and $R_{1} T_{1}^{\prime}$ satisfy the conditions of (1.4) of $\S 1$ and hence $R_{1} T_{1}=\operatorname{Re} S=R T$ and $R_{1} T_{1}^{\prime}=\operatorname{Im} S=R T^{\prime}$. Hence by Lemma $1, R_{1}^{2}=R^{2}$. Since $R_{1}$ commutes with $S, R_{1} E($. $)=E($. $) R_{1}$ by Theorem 5 of Dunford [2] and hence $R R_{1}=R_{1} R$. Let $A$ be a maximal commutative Banach subalgebra of $B(X)$ with identity, containing $R$ and $R_{1}$. Then the spectra of $R$ and $R_{1}$ are the same relative to $A$ and $B(X)$. Let $\mathfrak{M}$ be the space of maximal ideals of $A$. As $\sigma(R)=\sigma_{A}(R), \sigma(R)$ is the range of $R(M), M$ in $\mathfrak{M}$. Similarly, $\sigma\left(R_{1}\right)=$

2 The present simpler proof is due to the referee to whom the author is thankful. 
$\sigma_{A}\left(R_{1}\right)=$ the range of $R_{1}(M), M$ in $\mathfrak{M}$. Since $R^{2}=R_{1}^{2}$,

$$
\left(R^{2}-R_{1}^{2}\right)(M)=0
$$

for all $M$ in $\mathfrak{M}$. If for any $M,\left(R+R_{1}\right)(M)=0$, then $R(M)=-R_{1}(M)$. Since $\sigma(R)$ and $\sigma\left(R_{1}\right)$ are nonnegative, it follows that $R(M)=0=R_{1}(M)$. If $\left(R+R_{1}\right)(M) \neq 0$, then by $(2)\left(R-R_{1}\right)(M)=0$. Hence in all cases, $\left(R-R_{1}\right)(M)=0$. Since by (1.3), $R-R_{1}$ is hermitian in some equivalent norm, by a result of Vidav [9], it follows that $R-R_{1}=0$. Hence $R=R_{1}$. This completes the proof of the theorem.

CoRollary. If the space $X$ is a Hilbert space and $S$ is a normal operator on $X$, then

$$
S=R U=U R
$$

where $R$ is positive and $U$ is unitary in the usual Hilbert space terminology.

From the proof of the first part of the above theorem it is clear that the remark in $\$ 2$ can be strengthened to the following result. ${ }^{3}$

A scalar type operator $U$ on a Banach space $X$ is unitary under some equivalent norm on $X$ if and only if $\sigma(U)$ lies on the unit circle.

REMARK. If an operator $S$ on a reflexive Banach space $X$ has the polar decomposition $S=R U$ where $R$ and $U$ satisfy conditions (i) and (ii) of Theorem 1 , then $S$ is scalar type.

For, if $S=R U=R\left(T+i T^{\prime}\right)=R T+i R T^{\prime}$ then $R T$ and $R T^{\prime}$ commute and $(R T)^{m}\left(R T^{\prime}\right)^{n}\{m, n=0,1,2, \cdots\}$ are hermitian under some equivalent norm $\|\cdot \cdot\| \mid$ on $X$. Since $X$ is reflexive, by Theorem 4.1 of Berkson [1], the remark follows.

Thus the polar decomposition in Theorem 1 characterises scalar type operators on reflexive Banach spaces. But, however, this does not characterise scalar type operators on nonreflexive Banach spaces as is shown in the following counter-example.

Counter-example. The operators $T$ and $T^{\prime}$ on the nonreflexive Banach space of Kakutani's example in [6] have clearly nonnegative spectrum and as pointed out by Lumer [8], T, $T^{\prime}$ and $T+T^{\prime}$ are all hermitian together with $T^{n} T^{\prime m}(n, m=0,1,2, \cdots)$ in the given norm of the space. Hence by taking $T+T^{\prime}=S=R$ and $U=I$ in the

${ }^{3}$ This strengthened result was pointed out by Prof. W. G. Bade and Prof. Ralph S. Phillips in their report on the author's thesis, to whom the author's thanks are due. 
above remark, it is easily seen that conditions (i) and (ii) of Theorem 1 are satisfied by $R$ and $U$ under the given norm of the space. But $S=T+T^{\prime}$ is not scalar type as proved by Kakutani in [6].

4. One-parameter group of unitary operators. In this section we obtain an extension of Stone's theorem on one-parameter group of unitary operators in Hilbert spaces for reflexive Banach spaces when the group in question is uniformly continuous. ${ }^{4}$

Definition 6. Let $\left\{T_{t}\right\}(-\infty<t<\infty)$ be a family of operators on a Banach space, such that $T_{0}=I$ and $T_{t} T_{s}=T_{t+s}$. Then we call $\left\{T_{t}\right\}$ an one-parameter group of operators. It is said to be a uniformly continuous one-parameter group, if it further satisfies the condition that $t$ tends to $s$ implies that $T_{t}$ tends to $T_{s}$ in the uniform operator topology.

THEOREM 2. Let $U$ be a scalar type operator on a Banach space $X$, with its spectrum lying on the unit circle. Let $E($.$) be$ its resolution of the identity. Then the group

$$
\mathfrak{S} \equiv\left\{U_{t}=\int_{0}^{2 \pi} e^{i t \theta} F(d \theta) ;-\infty<t<\infty\right\}
$$

where

$$
F(\sigma)=E\left\{e^{i \theta}: \theta \in \sigma \cap(0,2 \pi]\right\}
$$

for Borel sets $\sigma$ of the complex plane, is a uniformly continuous one-parameter group of operators, all of them being unitary in some equivalent norm $|\|\cdot \mid\|$ on $X$.

Proof. The norm $\|\mid \cdot\|$ defined by

$$
\|x\|=\sup _{\| x^{*} \mid i=1} \operatorname{var} x^{*} E(.) x
$$

is an equivalent norm on $X$ and $E(\sigma)$ are hermitian in $\|\cdot\| \|$ for Borel sets $\sigma$ of the complex plane. That $\subseteq$ is an one-parameter group of operators is clear from the spectral properties of $U_{t}$. By arguing as in Theorem 1 in the case of $U$, it is easy to establish that $U_{t}$ are unitary in the norm $\||\cdot|\|$.

${ }^{4}$ A strongly continuous version of Stone's theorem for arbitrary Banach spaces has also been established in the author's $\mathrm{Ph}$. D. dissertation, which will appear elsewhere. The strongly continuous form of Stone's theorem for weakly complete Banach spaces has been obtained by Berkson (Semi-groups of scalar type operaters and a theorem of Stone, Illinois Journal of Mathematics, Vol. 10, pp. 345-352, 1966). But his method of proof is different. 
For real $t$ and $s$, by Lemma 6 of Dunford [2] we have,

$$
\begin{aligned}
\left\|U_{t}-U_{s}\right\| & =\left\|\left|\int_{0}^{2 \pi}\left(e^{i t \theta}-e^{i s \theta}\right) F(d \theta) \|\right|\right. \\
& \leqq 4 \mathrm{M} \sup _{\theta}\left|e^{i s \theta}\left(e^{i(t-s) \theta}-1\right)\right| \\
& \leqq 4 \mathrm{M} .2 \pi\left(e^{|t-s|}-1\right)
\end{aligned}
$$

so that $U_{t}$ tends to $U_{s}$ in the uniform operator topology, as $t$ tends to $s$. This completes the proof.

Conversely, suppose we are given a uniformly continuous oneparameter group of operators, all being unitary under some equivalent norm. Then is it possible to represent the group as in Theorem 2 ? Before answering this question in the affirmative in the case of reflexive Banach spaces, we shall presently discuss a necessary condition (condition $(H)$ below) for the converse of Theorem 2 to hold.

Definition 7. Let $\left\{T_{t}\right\} t \in A$ ( $A$-an indexing set) be a family of scalar type operators on a Banach space $X$. Let $R_{t}$ and $J_{t}$ denote the $\operatorname{Re} T_{t}$ and $\operatorname{Im} T_{t}$ respectively for all $t$. We say the family $\left\{T_{t}\right\}$ satisfies the condition $(H)$ under the equivalent norm $\|\cdot\|$ if $\|\cdot\|$ is an equivalent norm on $X$ such that

$$
R_{t_{1}} R_{t_{2}} \cdots R_{t_{i}} J_{s_{1}} J_{s_{2}} \cdots J_{s_{k}}
$$

are hermitian in $\|\cdot\|$ for all finite $i$ and $k$.

REMARK. To represent a group $\left\{T_{t}\right\}(-\infty<t<\infty)$ of scalar type operators in the form given in theorem 2 , it is necessary that $\left\{T_{t}\right\}$ satisfies the condition $(H)$ under some equivalent norm on $X$.

For, suppose

$$
T_{t}=\int_{0}^{2 \pi} e^{i t \theta} F(d \theta) \quad(-\infty<t<\infty)
$$

so that $F($.$) is the resolution of the identity of K=\int \theta F(d \theta)$. Then $F(\sigma)$ are hermitian in the norm defined by

$$
\|x\|=\sup _{\left\|x^{*}\right\|=1} \operatorname{var} x^{*} E(.) x
$$

and hence $\left\{T_{t}\right\}$ satisfies the condition $(H)$ under the norm \|\|$\cdot \|$.

Note that in a Hilbert space, a commuting family $\left\{T_{t}\right\}$ of normal operators automatically satisfies the condition $(H)$ in the given Hilbert space norm. But this is not true in a Banach space, even if the space is reflexive and $\left\{T_{t}\right\}, t \in A$, is a commuting family of scalar type 
operators with $F=\left\{\operatorname{Re} T_{t}, \operatorname{Im} T_{t} ; t \in A\right\}$ hermitian-equivalent. (Vide. $\S$ III of Lumer [8]).

To establish the converse of Theorem 2, we need the following lemma.

LEMma 2. Let $\left\{T_{t}\right\} t \in A$ be a commuting family of scalar type operators, satisfying the condition $(H)$ under the norm $\|\cdot\|$. Then the Banach algebra $B$ generated by $\left\{R_{t}=\operatorname{Re} T_{t}, J_{t}=\operatorname{Im} T_{t} ; t \in A\right\}$ and the identity operator $I$, in the uniform operator topology is commutative and every element $S$ of $B$ has the representation in the form $S=U+i V$ where $U$ and $V$ are in $B$, with $U$ and $V$ hermitian in $\|\cdot\|$. Under the involution defined by $S^{*}=U-i V, B$ is a Banach ${ }^{*}$-algebra and under the equivalent norm given by $\|S\|_{0}=\left\|S^{* 1 / 2} S\right\|^{r_{2}}$, $B$ is a $B^{*}$-algebra.

Proof. Let $D=I \cup\left\{R_{t}, J_{t} ; t \in A\right\}$. Since the generating family $D$ is a commuting family by Theorem 5 of Dunford [2], the Banach algebra $B$ is commutative. In view of Theorem 1 of Vidav [9], the hypothesis of the lemma and the fact that and the fact that $B$ is commutative, the lemma follows as soon as we establish that $S=U+i V$ for some $U$ and $V$ in $B$ with $U, V$ hermitian in $\|\cdot\|$. But this can be shown by an argument similar to that given by Berkson in Theorem 3.1 in [1]. This completes the proof.

THEOREM 3. (Extension of Stone's theorem). Let

$$
\mathfrak{S} \equiv\left\{U_{t} ;(-\infty<t<\infty)\right\}
$$

be a uniformly continuous one-parameter group of operators on a reflexive Banach space $X$, all being unitary under the equivalent norm \|\|$\cdot \|$ on $X$. Further, let $\subseteq$ satisfy the condition $(H)$ under this norm ||$\cdot \mid \|$. Then the group admits the unique spectral representation

$$
U_{t}=\int_{a}^{b} e^{i t \theta} E(d \theta),-\infty<t<\infty,
$$

for some bounded closed interval $[a, b]$ of the real line where the spectral measure $E($.$) commutes with every operator which commutes$ with the group $\mathfrak{S}$.

Proof. Let $R_{t}=\operatorname{Re} U_{t}, J_{t}=\operatorname{Im} U_{t}$ for all $t$ and

$$
D=\left\{R_{t}, J_{t} ;-\infty<t<\infty\right\} .
$$

Let $B$ be the Banach algebra generated by $D$ in the uniform operator 
topology.

Since $\mathfrak{S}$ satisfies the condition $(H)$ under the norm $\||\cdot|\|$ and $\subseteq$ is a commuting family of scalar type operators, by Lemma $2, B$ is a commutative Banach algebra and the Gelfand representation of $B$ is a bicontinuous isomorphism of $B$ onto $C(\mathfrak{M})$, where $\mathfrak{M}$ is the maximal ideal space of $B$. Hence each operator $T$ in $B$ corresponds to the function $T(m)$ in $C(\mathfrak{M})$ such that the correspondence is a bicontinuous isomorphism. ( $m$ denotes maximal ideals). Therefore, by Theorem 18 of Dunford [2], there exists a spectral measure $E_{1}($.$) on \mathfrak{M}$ to $B(X)$ such that

$$
T=\int_{\mathfrak{M}} T(m) E_{1}(d m)
$$

Hence in particular,

$$
U_{t}=\int_{\mathfrak{M}} U_{t}(m) E_{1}(d m)
$$

Since $\subseteq$ is a uniformly continuous one-parameter group of elements in $B$ by Theorem 9.4.2 of Hille and Phillips [5], there exists an element $a$ in $B$ such that

$$
U_{t}=e^{i t a} \quad \text { for }-\infty<t<\infty,
$$

where

$$
\mathfrak{a}=\frac{1}{i} \lim _{t \rightarrow 0} \frac{U_{t}-I}{t}
$$

Hence

$$
U_{t}(m)=e^{i t a(m)}, \quad-\infty<t<\infty .
$$

Let us denote $U_{1}$ by $U$. As the algebra $B$ is equivalent to $C(\mathfrak{M})$ the spectrum of $U$ in $B$ i.e. $\sigma_{B}(U)$ is the same as that of $U$ in $B(X)$; i.e. $\sigma(U)$. Therefore, $\sigma_{B}(U)$ is a subset of the unit circle. But $\sigma_{B}(U)$ is the range of $U(m)$ and hence $U(m)=e^{i \alpha(m)}$ has its range on the unit circle, so that $\mathfrak{a}(m)$ is real valued. Since $\mathfrak{a}(m)$ is a continuous function over the compact Hausdorff space $\mathfrak{M}$, its range is compact and hence is some bounded set $\tau$ of the reals. Let $[a, b]$ be some bounded closed interval containing $\tau$.

Since $\mathfrak{a}$ is in $B, \mathfrak{a}=\int_{\mathfrak{M}} \mathfrak{a}(m) E_{1}(d m)$. Hence by Lemma 6 of Dunford [2], $a$ is a scalar type operator with its resolution of the identity $E($.$) given by$

$$
E(.)=E_{1}\left(\mathfrak{a}^{-1}(.)\right)
$$

Hence 


$$
a=\int_{\tau} \lambda E\left(d \lambda_{\iota}\right)
$$

so that

$$
\begin{aligned}
U_{t} & =\int_{\mathfrak{M}} e^{i t a(m)} E_{1}(d m) \\
& =\int_{\tau} e^{i t \lambda} E(d \lambda) \\
& =\int_{a}^{b} e^{i t \theta} E(d \theta)
\end{aligned}
$$

since $[a, b] \supset \tau$.

The uniqueness of a follows from the equation (2) and as $\mathfrak{a}$ is a bounded scalar type operator its resolution of the identity is unique. Hence $E($.$) and therefore the spectral representation of \subseteq$ are unique.

To prove the last part of the theorem, let $T$ be any operator in $B(X)$ commuting with $\subseteq$. Since $T$ commutes with $U_{t}$ for all $t, T$ commutes with $R_{t}$ and $J_{t}$ for all $t$ by Theorem 5 of Dunford [2]; i.e. $T$ commutes with $D$. As $a$ is in $B$ and $B$ is the Banach algebra generated by $D, T$ commutes with $a$. But $a$ is a spectral operator with its resolution of the identity $E($.$) and hence again by Theorem$ 5 of Dunford [2], $T E()=.E()$.$T . This completes the proof of the$ theorem.

Note that the infinitesimal operator $a$ in the above theorem is hermitian in \|\|$\cdot \|$.

REMARK. If the Boolean algebra generated by the resolutions of the identities of $U_{t}$ for $-\infty<t<\infty$ is bounded, then by remarks in $\S$ III of Lumer [8] $\subseteq$ satisfies the condition $(H)$ under an equivalent norm on the Banach space $X$.

\section{REFERENCES}

1. E. Berkson, A characterisation of scalar type operators in reflexive Banach spaces, Pacific J. Math. 13 (1963), 365-373.

2. N. Dunford, Spectral operators, Pacific J. Math. 4 (1954), 321-354.

3. U. Fixman, Problems in spectral operators, Pacific J. Math. 9 (1959), 1029-1051.

4. S. R. Forguel, The relation between a spectral operator and its scalar part, Pacific

J. Math. 8 (1958), 51-65.

5. E. Hille and R. S. Phillips, Functional Analysis and Semi-groups, Amer. Math. Soc. Colloquium Publ., XXXI, 2nd ed., 1957.

6. S. Kakutani, An example concering uniform boundedness of spectral measures, Pacific J. Math. 4 (1954), 363-372.

7. G. Lumer, Semi-inner-product spaces, Trans. Amer. Math. Soc. 100 (1961), 29-43.

8. - Spectral operators, Hermitian operators and bounded groups, Acta. Sci. Math. (Szeged), 25 (1954), 75-85.

9. I. Vidav, Eine metrische Kennzeichnung der selbst-adjungierten Operatoren, Math.

Z. 66 (1956), 121-128. 
Received April 28, 1964, and in revised form October 4, 1966. This forms a slightly revised version of the Chapter II of the author's $\mathrm{Ph}$. D. dissertation (October, 1965) of the University of Madras, Madras. The author wishes to express his deep sense of gratitude to Professor V. K. Balachandran under whose guidance the dissertation was written.

UNIVERSITY OF MADRAS

MADRAS-5, INDIA

A. C. College of Technology

MADRAS-25, INDIA 



\section{PACIFIC JOURNAL OF MATHEMATICS}

\section{EDITORS}

\section{H. SAMELSON}

Stanford University

Stanford, California

J. P. JANS

University of Washington

Seattle, Washington 98105
J. DUGUNDJI

University of Southern California Los Angeles, California 90007

RICHARD ARENS

University of California

Los Angeles, California 90024

\section{ASSOCIATE EDITORS}
E. F. BECKENBACH
B. H. NEumanN
F. WOLF
K. YosidA

\section{SUPPORTING INSTITUTIONS}

\author{
UNIVERSITY OF BRITISH COLUMBIA \\ CALIFORNIA INSTITUTE OF TECHNOLOGY \\ UNIVERSITY OF CALIFORNIA \\ MONTANA STATE UNIVERSITY \\ UNIVERSITY OF NEVADA \\ NEW MEXICO STATE UNIVERSITY \\ OREGON STATE UNIVERSITY \\ UNIVERSITY OF OREGON \\ OSAKA UNIVERSITY \\ UNIVERSITY OF SOUTHERN CALIFORNIA
}

\author{
STANFORD UNIVERSITY \\ UNIVERSITY OF TOKYO \\ UNIVERSITY OF UTAH \\ WASHINGTON STATE UNIVERSITY \\ UNIVERSITY OF WASHINGTON \\ AMERICAN MATHEMATICAL SOCIETY \\ CHEVRON RESEARCH CORPORATION \\ TRW SYSTEMS \\ NAVAL ORDNANCE TEST STATION
}

Mathematical papers intended for publication in the Pacific Journal of Mathematics should be typewritten (double spaced). The first paragraph or two must be capable of being used separately as a synopsis of the entire paper. It should not contain references to the bibliography. Manuscripts may be sent to any one of the four editors. All other communications to the editors should be addressed to the managing editor, Richard Arens at the University of California, Los Angeles, California 90024.

50 reprints per author of each article are furnished free of charge; additional copies may be obtained at cost in multiples of 50 .

The Pacific Journal of Mathematics is published monthly. Effective with Volume 16 the price per volume ( 3 numbers) is $\$ 8.00$; single issues, $\$ 3.00$. Special price for current issues to individual faculty members of supporting institutions and to individual members of the American Mathematical Society: $\$ 4.00$ per volume; single issues $\$ 1.50$. Back numbers are available.

Subscriptions, orders for back numbers, and changes of address should be sent to Pacific Journal of Mathematics, 103 Highland Boulevard, Berkeley 8, California.

Printed at Kokusai Bunken Insatsusha (International Academic Printing Co., Ltd.), 7-17, Fujimi 2-chome, Chiyoda-ku, Tokyo, Japan.

PUBLISHED BY PACIFIC JOURNAL OF MATHEMATICS, A NON-PROFIT CORPORATION

The Supporting Institutions listed above contribute to the cost of publication of this Journal, but they are not owners or publishers and have no responsibility for its content or policies. 


\section{Pacific Journal of Mathematics

Vol. 22, No. $3 \quad$ March, 1967

Wai-Mee Ching and James Sai-Wing Wong, Multipliers and $H^{*}$

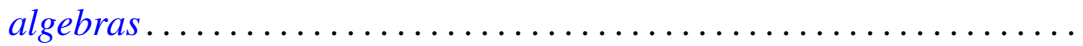

P. H. Doyle, III and John Gilbert Hocking, A generalization of the Wilder

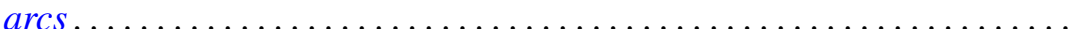

Irving Leonard Glicksberg, A Phragmén-Lindelöf theorem for function

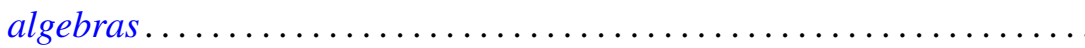

E. M. Horadam, A sum of a certain divisor function for arithmetical semi-groups ..................................... 407

V. Istrăţescu, On some hyponormal operators ................... 413

Harold H. Johnson, The non-invariance of hyperbolicity in partial

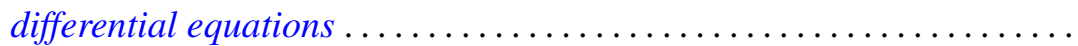

Daniel Paul Maki, On constructing distribution functions: A bounded denumerable spectrum with $n$ limit points................... 431

Ronald John Nunke, On the structure of Tor. II .................... 453

T. V. Panchapagesan, Unitary operators in Banach spaces ............. 465

Gerald H. Ryder, Boundary value problems for a class of nonlinear differential equations ................................. 477

Stephen Simons, The iterated limit condition and sequential convergence .................................... 505

Larry Eugene Snyder, Stolz angle convergence in metric spaces ......... 515 Sherman K. Stein, Factoring by subsets ................... 523

Ponnaluri Suryanarayana, The higher order differentiability of solutions of abstract evolution equations . . .

Leroy J. Warren and Henry Gilbert Bray, On the square-freeness of Fermat and Mersenne numbers ............................... 563

Tudor Zamfirescu, On l-simplicial convexity in vector spaces........... 565 Eduardo H. Zarantonello, The closure of the numerical range contains the spectrum 